\title{
Age-related changes in the trachea in healthy adults.
}

\section{AUTHOR(S):}

Sakai, Hiroaki; Nakano, Yasutaka; Muro, Shigeo; Hirai, Toyohiro; Takubo, Yasutaka; Oku, Yoshitaka; Hamakawa, Hiroshi; ... Hirata, Toshiki; Date, Hiroshi; Mishima, Michiaki

\section{CITATION:}

Sakai, Hiroaki ... [et al]. Age-related changes in the trachea in healthy adults.. Advances in experimental medicine and biology 2010, 662(Part 1): 115-120

\section{ISSUE DATE:}

2010

URL:

http://hdl.handle.net/2433/128869

\section{RIGHT:}

The original publication is available at www.springerlink.com; This is not the published version. Please cite only the published version.; この 論文は出版社版でありません。引用の際には出版社版をご確認ご利用 ください。 


\title{
Age-related changes in the trachea in healthy adults
}

\author{
Hiroaki Sakai $^{1}$, Yasutaka Nakano ${ }^{6}$, Shigeo Muro ${ }^{2}$, Toyohiro Hirai ${ }^{2}$, Yasutaka \\ Takubo $^{7}$, Yoshitaka Oku ${ }^{5}$, Hiroshi Hamakawa ${ }^{3}$, Ayuko Takahashi ${ }^{3}$, \\ Toshihiko Sato $^{4}$, Fengshi Chen ${ }^{3}$, Hisashi Sahara', Takuji Fujinaga ${ }^{3}$, Kiyoshi \\ Sato $^{1}$, Makoto Sonobe ${ }^{1}$, Tsuyoshi Shoji ${ }^{1}$, Ryo Miyahara ${ }^{1}$, Kenichi Okubo ${ }^{1}$, \\ Toru Bando $^{3}$, Toshiki Hirata ${ }^{1}$, Hiroshi Date ${ }^{1}$ and Michiaki Mishima ${ }^{2}$ \\ Departments of ${ }^{1}$ Thoracic Surgery, ${ }^{2}$ Respiratory Medicine and ${ }^{3}$ Organ Preservation \\ Technology, Graduate School of Medicine, ${ }^{4}$ Department of Bioartificial organs, Institute for \\ Frontier Medical Sciences, Kyoto University, Kyoto, 606-8507, Japan, ${ }^{5}$ Department of \\ Physiology, Hyogo college of medicine, Nishinomiya, 663-8501, Japan ${ }^{6}$ Division of \\ Respiratory Medicine, Department of Medicine, Shiga University of Medical Science, Otsu, \\ 520-2192, Japan, ${ }^{7}$ Hamamatsu Rosai Hospital, Hamamatsu, 430-8525, Japan.
}

\begin{abstract}
To investigate age-related changes in the shape of trachea, normal male volunteers $(\mathrm{n}=83$, mean $\pm \mathrm{SD}$ : $47.7 \pm 20.2$ years old) underwent inspiratory CT scans at full inspiration and lung function tests. Subjects who showed $\mathrm{VC}<80 \%$ predicted or FEV1 $<80 \%$ predicted on lung function tests were excluded. The CT data, which is located at $2.0 \mathrm{~cm}$ above the aortic arch, were transferred to a personal computer. The tracheal area (St) and two parameters, Tracheal index (Ti) and Circularity (Ci) indicating the shape of the trachea, were automatically calculated. Ti was defined the ratio of the coronal to the sagittal diameter of the trachea, and the $\mathrm{Ci}(\mathrm{Ci}=4 \pi \mathrm{S} / \mathrm{L} 2$, S: tracheal area, $\mathrm{L}$ : tracheal perimeter) was used to indicate the roundness of the trachea. A Ci value of less than 1 indicated the distortion of the roundness. Both St and St/BSA (body surface area) showed a significant correlation with age $(\mathrm{r}=0.37, \mathrm{r}=0.52 ; \mathrm{p}=0.0006, \mathrm{p}<0.0001)$. Ti was not correlated with age $(\mathrm{r}=-0.20 ; \mathrm{p}=0.0697)$, whereas $\mathrm{Ci}$ was significantly correlated with age $(\mathrm{r}=-0.32 ; \mathrm{p}=0.00364)$. There were measurable age related changes of the trachea both in the area and the shape. Aging results in the increased tracheal area and a distortion of the roundness.
\end{abstract}




\section{Introduction}

The automated method for the analysis of the digital computed tomography (CT) images has been developed recently $[3,4,7,8,9]$. Using CT images, we can evaluate the airway size and configuration on its transection plane in vivo [10], and can examine in detail compared to plain chest roentgenogram. For example, we have reported that the trachea was distorted in COPD patients [21]. Hoffmann et al. showed the change in the tracheal cross-sectional area in children from 0-20 years old by CT image [22]. However, to our knowledge, there was no report about the age-related changes of the tracheal configuration evaluated by CT in healthy adults. In this study, we used an automated method for the analysis of the tracheal lumen on its size and configuration, and investigated age-related changes of those parameters.

\section{Methods}

This study included eighty-three healthy normal subjects ranging from 21 to 83 years old who were specially recruited for this study. They had no history of asthma or serious previous chest disease. Approval for the study was obtained from the Ethics Committee of Kyoto University, and written informed consent was obtained from each subject prior to the study. Pulmonary function was tested with a Chestac-65V (Chest MI, Tokyo, Japan) on the same day that CT scans were performed. Vital capacity (VC), forced expiratory flow in one second (FEV1), residual volume (RV) by helium dilution method, and carbon monoxide diffusing capacity (DLCO) by the single breath method were measured. Subjects who showed VC $<80 \%$ predicted or FEV1 $<80 \%$ predicted on lung function tests were excluded. CT scans were performed with the subject in the supine position using the high-resolution scanner, X-Vigor (Toshiba, Tokyo, Japan), with the following settings: $2 \mathrm{~mm}$ slice thickness, scanning time $1.0 \mathrm{~s}$, electrical voltage 120 $\mathrm{kV}$, electrical current $200 \mathrm{~mA}$, and a field of view (FOV) $32 \mathrm{~cm}[6,7,8]$. Standard reconstruction algorithm for the lung field (FC85) was adopted. During the scan, subjects were requested to hold his breath after deep inspiration. Contrast medium was not used. Each CT image was composed of 512 x 512 matrix data of Hounsfield Unit (HU). The CT data were transferred to a personal computer, and were analyzed using custom software. The software automatically detected lung fields $[3,9]$, and trachea [21]. Tracheal size was analyzed on the CT image at $2 \mathrm{~cm}$ above the upper margin of the aortic arch. When the tracheal lumen was detected, the area, the sagittal length (Lsag) and the coronal length (Lcor) of the tracheal lumen were automatically measured. The short radius (SR) was defined as the minimal distance from the centroid of the tracheal lumen to the internal surface of the tracheal wall, and the long radius (LR) was defined as the maximum distance. 
The tracheal index (Ti) was defined as the ratio of Lcor/Lsag for the index of tracheal deformity $[1,2]$. We also calculated another index to evaluate the roundness of the trachea; circularity (Ci) as $4 \pi \mathrm{Str} /$ a square of internal perimeter [21]. In general, if the figure is the circle, circularity is one. When the figure is distorted from circle, the circularity decreases. Therefore, circularity is the parameter for the roundness. All data were expressed mean $\pm \mathrm{SD}$. Linear regression analysis was used to determine the correlation between age and the results of the parameter for tracheal configuration, Fischer's Z transform test was used for to evaluate a significant correlation with age. A p value $p<0.05$ was considered to be statistically significant.

\section{Results}

Table 1 shows the results of the size and parameters for configuration of the trachea. Table 2 shows the correlation coefficients between age and the parameters of the trachea. Both area of trachea (Str) and Str/BSA (body surface area) showed a significant correlation with age $(\mathrm{r}=0.34, \mathrm{p}=0.0069 ; \mathrm{r}=0.50, \mathrm{p}<0.0001)$. However, Ti was not correlated with age, whereas both $\mathrm{Ci}$ and Lsag were significantly correlated with age $(r=-0.27, p=0.0037 ; r=0.42, p<0.0001)$.

\section{Discussion}

The results of this study showed that there were measurable age-related changes in the trachea both in the area and the shape. Significant information concerning tracheal cross-section has been observed from the diameter on frontal or lateral chest radiographs [23]. However, variations in the shape of the trachea and locations not tangential to the x-ray beam can complicate such analysis. Tsao and Shich [10] reported that Lsag of the trachea had a significant positive linear correlation with lateral chest diameter, and Trigaux et al. [11] showed that the Ti was significantly correlated with the functional residual capacity and with the sternum-spine distance. In this study, we used CT to more accurately evaluate the size and the shape of the trachea. Although Ti measured by CT failed to show significant relationship with RV/TLC, Ci, Str/BSA and Lsag correlated significantly with RV/TLC. More importantly, Ci, Lsag, Str, Str/BSA correlated with age.

Rains et al. showed that there was a progressive increase in the tensile stiffness of tracheal cartilage with age. James et al. [12] .and Moreno et al. [13, 14] have shown that the degree of shortening of maximally contracted airway smooth muscle is influenced by the integrity of airway cartilage. The increased cartilage stiffness with increasing age could increase resting smooth muscle tension and de- 
crease the potential for smooth muscle shortening by causing an increased after load. And since the shape of the trachea might be influenced by both sides of the lung, it is reasonable to get the result that the sagittal length of the trachea is increasing.

We also found that the measurements of the trachea correlated with FEV1. Airway cartilage is mechanically important in resisting airway collapse during maximal expiratory flow maneuvers and cough. The wave-speed theory of maximal expiratory flow limitation predicts that maximal expiratory flow from the lung is determined not only by the driving pressure and the resistance of airways upstream from the flow-limiting segment but also by the cross-sectional area and compliance of the airway at the flow-limiting site [15]. Studies both in humans [16, 17] and in experimental animals [18] show that the flow-limiting sites within the tracheobronchial tree are located predominantly in central cartilaginous airways during most of the forced expiratory vital capacity. Because cartilage makes up such an important structural component of the airway wall in central airways, it is reasonable to assume that its mechanical properties are important in determining their pressure-area behavior. Caldwell and Fry [19] and McCormack et al. [20] have shown that softening of tracheal cartilage by the intravenous injection of papain in the rabbit results in a significant reduction in maximal expiratory flow from the lung. In the present study we have found a significant negative correlation between age and PFR $(r=0.637, \mathrm{P}=0.00037)$. The Ti was originally defined as the ratio of Lcor, measured on the lateral view of a plain roentgenogram, to Lsag, measured on the posteroanterior view [1]. On plain roentgenograms, however, the length on the image is not the same as the real value because the image is enlarged in accordance with the distance between the subject and the film. Therefore, we used the $\mathrm{CT}$ image to make a precise evaluation of the trachea. On CT images, the axis of the trachea, however, is not always parallel to the sagittal plane nor to the horizontal plane. The plane of the CT image may not be vertical to the long axis of the trachea, and we may overestimate the deformity of the trachea on CT images. Therefore, we calculated the centroid of the trachea, using two CT images ( 2 and 4 $\mathrm{cm}$ above the top of the aortic arch) and determined the long axis of the trachea. Then, we calculated the angle of deviation between the long axis of the trachea to the vertical axis of the CT plane as previously reported [21].

The importance of this study is that the shape of the trachea is gradually distorted and increased in size by aging. When we consider the aging the residual volume is increasing with age, and this effect is more sensitive to the airway size. From the physiological view point increase in the size of the trachea with age is an acceptable result for the decrease in the flow-limitation with age. We concluded that there were measurable age-related changes in the trachea both in the area and the shape. Aging may result in the increased tracheal area and a distortion of the roundness.

Acknowledgments The authors thank Arnab Majumdar of the Department of Physics, Boston University for helpful advice and manuscript editing. 


\section{References}

[1] Greene R et al: 'Saber-sheath' trachea: Relation to chronic obstructive pulmonary disease. Am J Roentgenol 1978; 130:441- 445.

[2] Greene R et al: 'Saber-Sheath' trachea: A clinical and functional study of marked coronal narrowing of the intrathoracic trachea. Radiology 1975; 115:258-265.

[3] Sakai $\mathrm{N}$ et al: An automated method to assess the distribution of low attenuation areas on chest CT scans in chronic pulmonary emphysema patients. Chest 1994; 106: 1319-1325.

[4] Nakano Y et al: Comparison of low attenuation areas on CT between inner and outer segments of the lung in COPD patients: Incidence and contribution to lung function. Thorax 1999; 54: 384-389.

[5] Kory RC et al: The Veterans Administration-Army Cooperative Study of Pulmonary Function. I. Clinical spirometry in normal men. Am J Med 1961;30: 243-258.

[6] Nishimura $\mathrm{K}$ et al: Comparison of different computed tomography scanning methods for quantifying emphysema. J Thorac Imaging 1998;13:193-198.

[7] Mishima $\mathrm{M}$ et al: Optimized scanning conditions of HRCT in the follow-up of pulmonary emphysema. J Comput Assist Tomogr 1999;23:380-384.

[8] Mishima M et al: Complexity of terminal airspace geometry assessed by lung computed tomography in normal subjects and patients with chronic obstructive pulmonary disease. Proc Natl Acad Sci USA 1999;96:8829-8834.

[9] Dechman G et al: Assessment of acute pleural effusion in dogs by computed tomography. J Appl Physiol 1994;76: 1993-1998.

[10] Tsao TC and Shieh WB: Intrathoracic tracheal dimensions and shape changes in chronic obstructive pulmonary disease. Taiwan I Hsueh Hui Tsa Chih 1994;93:30-34.

[11] Trigaux JP et al: CT of saber-sheath trachea. Correlation with clinical, chest radiographic and functional findings. Acta Radiol 1994; 35:247-250.

[12] James AL et al: Quantitative measurement of smooth muscle shortening in isolated pig trachea. J. Appl. Pysiol. 1987; 63: 1360-1365.

[13] Moreno RH et al: Isovolumetric and isobaric rabbit tracheal contraction in vitro. J. Appl. Physiol. 1987:62: 82-90.

[14] Moreno RH et al: Intravenous papain-induced cartilage softening decreases preload of tracheal smooth muscle. : Appl. Physiol. 1989; 66: 1694-1698.

[15] Dawson SV et al: Wave-speed limitation on expiratory flow-a unifying concept. J. Appl. Physiol. 1977;43: 498-515.

[16] Fraser, RG et al: Measurement of caliber of the human bronchi in 3 phases of respiration by tine bronchography. J. Can. Assoc. Radiol. 1961 ; 31: 102-110,1961.

[17] Fraser, RG et al: Bronchial dynamics in health and obstructive airway disease: physiology and roentgenology. In: Frontiers of Chest Radiology, edited by M. Simon, E. J. Potchen, and N. LeMay. New York: Grume \& Stratton, 1969, p. 76-101.

[18] Mink S et al: Mechanism of increased maximum expiratory flow during $\mathrm{HeO}$, breathing in dogs. J. Appl. Physiol. 1979; 47: 490-502.

[19] Caldwell EJ et al: Pulmonary mechanics in the rabbit. J. Appl. Physiol. 1969; 27: 280-285.

[20] Mccormack GS et.al: Lung mechanics in papain treated rabbits, J. Appl. Physiol. 1986; 60:242-246.

[21] Muro S et.al: Distorted Trachea in Patients with Chronic Obstructive Pulmonary Disease. Respiration 2000;67:638-644.

[22] Eoffmann EL et.al: Tracheal Cross-Sectional Area in Children: CT Determination. Radiology $1983 ; 149: 137-140$

[23] Mishima M et.al: Standardization of low attenuation area versus total lung area in chest Xray CT as an indicator of chronic pulmonary emphysema. Front Med Biol Eng 1997;8:79-86. 
Table 1. The parameters for the tracheal size and configuration in normal subjects

\begin{tabular}{|c|c|c|c|c|c|}
\hline & & Mean Value & SD & Minumum & Maximum \\
\hline $\mathrm{Ci}$ & & \begin{tabular}{|l|}
0.87 \\
\end{tabular} & 0.03 & 0.75 & 0.91 \\
\hline $\mathrm{Ti}$ & & 0.91 & 0.11 & 0.59 & 1.34 \\
\hline Lsag & $(\mathrm{mm})$ & 20.2 & 2.5 & 16.4 & 28.5 \\
\hline Lcor & $(\mathrm{mm})$ & 18.2 & 2.01 & 14.6 & 26.3 \\
\hline Str & $\left(\mathrm{mm}^{2}\right)$ & 287.4 & 56 & 188.7 & 520.8 \\
\hline Str/BSA & & 166.9 & 35.5 & 110.1 & 302.9 \\
\hline
\end{tabular}

Ci: Circularity, Ti: Tracheal Index (Lcor/Lsag), Lsag: sagittal length, Lcor: coronal length, Str: area of trachea, BSA: Body Surface Area

Table 2. Correlation coefficients of parameters for tracheal configuration versus age in normal subjects

\begin{tabular}{|c|c|c|}
\hline \multicolumn{2}{|c|}{ correlation coefficien } & $\mathrm{p}$ value \\
\hline $\mathrm{Ci}$ & -0.32 & $0.00364^{*}$ \\
\hline $\mathrm{Ti}$ & -0.2 & 0.0697 \\
\hline $\mathrm{Lsag}$ & 0.41 & $<.0001^{*}$ \\
\hline $\mathrm{Lcor}$ & 0.21 & 0.0629 \\
\hline $\mathrm{Str}$ & 0.37 & $0.0006^{*}$ \\
\hline Str/BSA & 0.52 & $<.0001^{*}$ \\
\hline
\end{tabular}

Ci: Circularity, Ti: Tracheal Index (Lcor/Lsag), Lsag: sagittal length, Lcor: coronal length, Str: area of trachea, BSA: body surface area $\quad *: p<0.05$ (Fisher's $Z$ transform ) 